\title{
Aspectos motivacionais relacionados à prática do método pilates
}

\author{
Cristina Carvalho de Melo* \\ Franco Noce** \\ Wagner Jorge dos Santos*** \\ Michelle Sena de Castro Silva**** \\ Jackson Martins Filho***** \\ Herbert Ugrinowitsch******
}

\section{Resumo}

O presente estudo objetiva identificar os aspectos motivacionais que levam os indivíduos a praticar o Método Pilates. Foi aplicado o questionário IMPRAFE-54 em 52 indivíduos adultos e idosos. Variáveis como sexo, faixa etária e tempo de prática foram consideradas. Para as comparações de grupos foram realizados teste T e Mann-Whitney. Os resultados mostraram que, de uma forma geral, a amostra se motiva pelo fator saúde seguido pelo prazer. A variável saúde apresentou diferença significativa entre idosos e adultos. Mulheres adultas se motivam mais pelo domínio estética quando comparadas às idosas. Homens adultos se motivam mais pelo domínio saúde quando comparados aos idosos. Entender os aspectos motivacionais relacionados à prática do Método Pilates pode contribuir para uma melhor intervenção diretamente associada à satisfação dos praticantes.

Palavras-chave: Pilates, Motivação, Aspectos Psicológicos.

\section{Motivational aspects related to the practice of the pilates method}

\begin{abstract}
This study aims to identify the motivational aspects that lead individuals to practice the Pilates Method. The IMPRAFE-54 questionnaire was applied to 52 adult and elderly individuals. Variables such as sex, age group and time of practice were considered. For group comparisons, the T test and the Mann-Whitney test were performed. The results showed that, in general, the sample is motivated by the health factor followed by pleasure. The health variable showed a significant difference between the elderly and adults. Adult women are more motivated by the aesthetic domain when compared to the elderly. Adult men are more motivated by the health domain when compared to the elderly. Understanding the motivational aspects related to the practice of the Pilates Method can contribute to a better intervention directly associated with the satisfaction of practitioners.
\end{abstract}

Keywords: Pilates, Motivation, Psychological aspects.

* Doutora em Ciências do Esporte, Mestre em Estudos do Lazer e Graduada em Educação Física pela UFMG. Idealizou e Coordena o Projeto PILATES UFMG.

** Doutor em Psicobiologia pela UNIFESP/EPM, Mestre e Graduado em Educação Física pela UFMG. Psicólogo pela Faculdade Pitágoras. Prof. Efetivo do PPG em Ciências do Esporte - UFMG. Vice-Presidente da Associação de Estudos em Psicologia do Esporte e do Exercício ABEPEEx

***Doutorado e Pós-Doc em Saúde Coletiva pela FIOCRUZ. Graduado em Psicologia pela PUC/MG e em Educação Física pelo Centro Universitário-MG. ****Fisioterapeuta pelo UNI/BH e Mestranda em Ciência Reabilitação - UFMG.

***** Graduado em Educação Física pela UFMG.

******Graduado em Educação Física pela FEFISA. Doutor e Mestre pela USP/SP. Prof. da UFMG e Vice-Presidente da Sociedade Brasileira de Comportamento Motor. Pós-Doutorado pela University of Queensland - Austrália. 


\section{Introdução}

A Organização Mundial de Saúde (OMS) mostra que o sedentarismo é o quarto principal fator de risco de morte no mundo, sendo um elevado fator de risco para doenças crônicas não transmissíveis, como diabetes, câncer e doenças cardiovasculares (OMS, 2017). Um estilo de vida ativo pode conduzir a ganhos significativos para saúde e prevenção de diversas doenças.

Existe uma campanha da OMS para redução de 25\% da mortalidade causada pelas doenças crônicas não transmissíveis e redução de $10 \%$ na inatividade física até 2025 , com plano de ação global para promoção da saúde através do incentivo à prática de exercícios físicos (OMS 2018).

A procura por exercícios físicos vem crescendo no Brasil, houve um aumento de 30,3\% em 2009 para 37,6\% de indivíduos buscando práticas esportivas em 2016 (Vigitel, 2017). Devido à veiculação, na mídia, dos benefícios que o exercício físico proporciona, verifica-se um aumento do número de pessoas de diversas faixas etárias e de ambos os sexos que procuram esta prática com intuito de prevenção de doenças, bem-estar, melhora da condição física, prazer (Legnani, Guedes, Legnani, Barbosa Filho \& Campos, 2011), qualidade de vida e fatores estéticos (Tahara, Schwartz \& Silva 2003), bem como evitar o sedentarismo. Para contribuir com esta perspectiva a adoção de hábitos saudáveis como a prática de exercícios físicos, temos o Método Pilates. A evolução e a popularização do método de Pilates andam na mesma direção da busca por sustentabilidade e qualidade de vida (Zen, Rempel \& Grave, 2016). No Brasil o método Pilates tem sido utilizado tanto para reabilitação, quanto para condicionamento físico (Liposcki, Ribeiro \& Schneider, 2016).

O idealizador do Método Pilates, Joseph H. Pilates nasceu na Alemanha em 1883 e iniciou o desenvolvimento do método em 1914, em um campo de refugiados, durante a I Guerra mundial. Utilizando molas de cama de hospital e outros materiais, construiu a base dos mesmos aparelhos que são utilizados hoje em dia no mundo todo. Através dos equipamentos desenvolvidos, ele ensinou a prática de um método de condicionamento físico e exercícios para seus companheiros ajudando-os a sobreviver à pandemia de 1918 (Panelli \& De Marco, 2006). Nos anos posteriores a sua criação, o método Pilates foi expandido pelo mundo após a abertura do estúdio de Joseph em Nova York- EUA, chegando ao Brasil no ano de 1991. O método tem como base a contrologia, sistema de coordenação entre corpo mente e espírito, fundamentada em seis princípios: respiração, concentração, centramento (Power House), fluidez, precisão e controle dos movimentos (Silva
\& Guerino, 2019; Branco, Miyamoto, Soliano, Farhat, Franco \& Cabral, 2017; Herrera-Gutiérrez, Olmos-Soria, \& Brocal-Pérez, 2015).

Alguns dos benefícios com a prática do método Pilates descritos na literatura, são: melhora de dores lombares (Schossler, Valente, Bittencourt \& Strassburger, 2013), melhora da flexibilidade e aumento da estabilidade articular (Barra \& Araújo, 2017; Branco, Miyamoto, Soliano, Farhat, Franco \& Cabral (2017), melhora da postura, equilíbrio, reeducação postural e redução dos níveis de estresse (Pinton \& Franco, 2007; Vieira, Testa, Ruas, Salvani, Catai \& Melo, 2017; Herrera-Gutiérrez, Olmos-Soria, \& Brocal-Pérez, 2015), redução de ansiedade (Herrera-Gutiérrez, Olmos-Soria, \& Brocal-Pérez, 2015), ganhos positivos na força muscular abdominal (Formighieri, 2012), melhora do desempenho no trabalho (Gaskell \& Williams, 2018), melhoria da qualidade de vida geral em adultos e idosos (Reis, Mascarenhas e Lyra, 2011; Silva \& Guerino, 2019), melhora do sono, concentração (Aliev \& Memmedora, 2015) bem como a melhoria da capacidade para realização das atividades de vida diária dos praticantes (Jesus, Cruz, Guedes, Legnanini, Barbosa Filho \& Campos, 2013; Pereira, Liberali, Lopes, Cruz, Artaxo Neto, Viana \& Mota, 2013; Gaskell \& Williams, 2018).

Ao buscar um exercício físico o indivíduo é impulsionado por motivos específicos que constroem sua motivação para a prática. A motivação pode ser caracterizada como o processo de mobilizar necessidades preexistentes relacionadas a fatores pessoais (intrínsecos) e ambientais (extrínsecos), bem como os tipos de comportamento capazes de satisfazê-las (Feijó, 1998). A motivação intrínseca retrata um caráter inconsciente, que representa o desejo interior de cumprir as tarefas propostas independentemente das condições externas, caracterizada pela força psíquica que leva o indivíduo a se empenhar em determinada atividade por vontade própria. Já a motivação extrínseca tem a característica de ser consciente e é definida pelos fatores externos que podem conduzir o comportamento do sujeito (Miranda \& Bara Filho, 2008). A prática de Pilates exerce influência intrínseca ao praticante, pois exige concentração e interiorização, proporcionando efeitos como: melhora de humor e do estado psicológico, redução de estresse e ansiedade, que gera maior satisfação interna (Aliev \& Memmedora, 2015).

Diante desses fatores motivacionais intrínsecos e extrínsecos, diversas variáveis assume um papel importante na escolha de um exercício físico, entre elas a faixa etária. Freitas, Santiago, Viana, Leão \& Frayre (2007) eviden- 
ciam que o público idoso opta por um exercício físico cuja socialização está intimamente presente nas práticas. Através de um estudo exploratório com praticantes em academias de ginástica, Balbinotti e Capozzoli (2008) obtiveram o resultado de que o púbico adolescente é mais motivado em relação ao prazer que a prática proporciona, enquanto jovens adultos e adultos de meia idade apresentam maior motivação pelo viés da saúde. Ainda nesse trabalho fica marcante que as mulheres aderem mais à prática de exercícios físicos que os homens quando os motivos são relacionados à saúde, evidenciando assim que a variável "sexo" também pode impactar na escolha de determinada prática.

Assim como a busca por outras atividades está relacionada à motivação do indivíduo, ao procurar o Pilates, em geral o indivíduo possui uma motivação específica. Motivação é o processo que guia o ser humano a se manter firme em um propósito, com objetivos determinados e envolve o contexto biológico, emocional, social e cognitivo (Aliev \& Memmedora, 2015). A revisão de literatura foi realizada por meio da busca de trabalhos relacionados ao Método Pilates com os descritores motivação e/ou fatores motivacionais. Há evidências escassas na literatura sobre os aspectos motivacionais relacionados à prática dessa modalidade, bem como estudos relacionados a outros aspectos psicológicos. Refletindo sobre a importância de se identificar a motivação dos indivíduos para a procura e permanência nas aulas de Pilates, surgiu a pergunta que direcionou o estudo: O que leva as pessoas a praticarem Pilates? Diante da escassez de pesquisas, do aumento da procura pela prática desse exercício físico e dos amplos benefícios descritos na literatura, o objetivo deste estudo foi identificar os aspectos motivacionais que levam os indivíduos a praticar o Método Pilates.

Material e Método

O presente estudo foi submetido à aprovação do Comitê de Ética em pesquisa da Universidade Federal de Minas Gerais e aprovado sob o número 30783914.3.0000.5149.

Todos os indivíduos foram informados previamente sobre a condução da pesquisa e concordaram com suas condições assinando o Termo de Consentimento Livre e esclarecido (TCLE), que é uma obrigatoriedade do conselho nacional de saúde (CNS), resolução no 466/12 em pesquisa envolvendo seres humanos.

Sujeitos

Participaram do estudo 52 alunos, sendo 11 homens ( 1 idoso e 10 adultos) e 41 mulheres (5 idosas e 36 adultos) com idade variando entre 19 e 70 anos, todos devidamente matriculados e ativos em um projeto de extensão universitária de Pilates. Todos os voluntários frequentavam as aulas de Pilates regularmente, duas vezes por semana.

\section{Procedimentos}

As coletas foram realizadas na sala do Projeto Pilates. As aplicações dos questionários ocorreram antes das aulas do projeto, entre $7 \mathrm{~h} 00 \mathrm{~min}$ e $20 \mathrm{~h} 00 \mathrm{~min}$, antes do início das aulas de Pilates.

Aos participantes, foi esclarecido como deveriam ser preenchidos os questionários. Os dados foram coletados em espaço padronizado para todos os participantes.

\section{Critérios de inclusão}

Estar matriculado e frequente nas aulas de Pilates proporcionadas pelo projeto de extensão universitária em Pilates.

\section{Critérios de exclusão}

Indivíduos que, por algum motivo não responderam todas as perguntas do questionário.

\section{Instrumento}

Para verificação da motivação, foi aplicado o questionário Escala de Motivação à Prática de exercícios físicos (IMPRAFE-54) com questões avaliadas a partir de uma escala tipo Likert variando de 1 a 5, sendo: 1- motiva pouquíssimo, 2- motiva pouco, 3- motiva moderadamente, 4- motiva muito e 5- motiva muitíssimo. O questionário apresenta 54 perguntas distribuídas em seis diferentes domínios: Controle do estresse, Saúde, Sociabilidade, Competitividade, Estética e Prazer (Barbosa, 2006).

\section{Análise Estatística}

A análise estatística foi realizada através do programa estatístico SPSS for Windows versão 22.0. Foi realizado um teste Shapiro-Wilk para verificar se as variáveis possuíam distribuição normal. Para comparar os grupos de acordo com a faixa etária (idoso $\geq 60$ anos e adultos $<60$ anos) e sexo foi realizado o teste T para as variáveis com distribuição normal e o teste de Mann-Whitney para as variáveis que não apresentavam distribuição normal.

\section{Resultados}

Dos cinquenta e dois participantes da pesquisa, $78,84 \%$ eram do sexo feminino. $\mathrm{Na}$ caracterização da amostra, a faixa etária se encontra entre 19 e 70 anos, 
a predominância $(36,53 \%)$ da idade dos voluntários se encontra entre 30 e 39 anos, sendo que o grupo idoso representa $11,53 \%$ do total de indivíduos estudados.

Já em relação ao tempo de prática do método de treinamento Pilates, a amostra apresentou uma variação de 0,5 a 120 meses de tempo de permanência na modalidade, no qual a predominância estava centrada no grupo entre 13 a 24 meses $(38,46 \%)$.

Em relação à escolaridade, a maioria da amostra $(96,15 \%)$ relatou ter concluído o ensino superior.

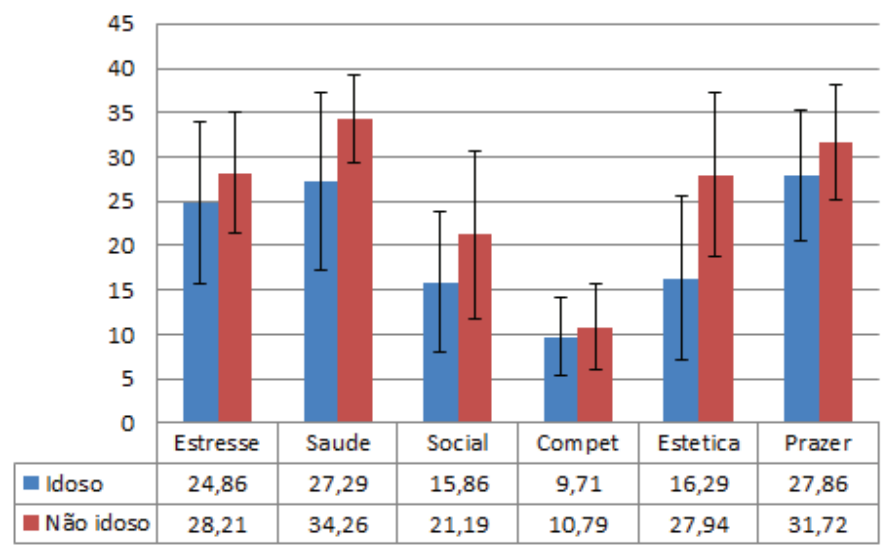

Figura 1. Comparação da motivação entre idosos e adultos

A figura 1 mostra que na comparação geral da motivação entre os idosos e adultos, não houve diferença significativa. A população não idosa apresentou escores maiores em relação aos domínios da saúde seguida pelo prazer. E, no enquanto a população idosa obteve escores maiores relacionados à variável prazer seguidos pela saúde. Observou-se que a população idosa apresenta escores inferiores em relação à estética quando comparado com a população não idosa.

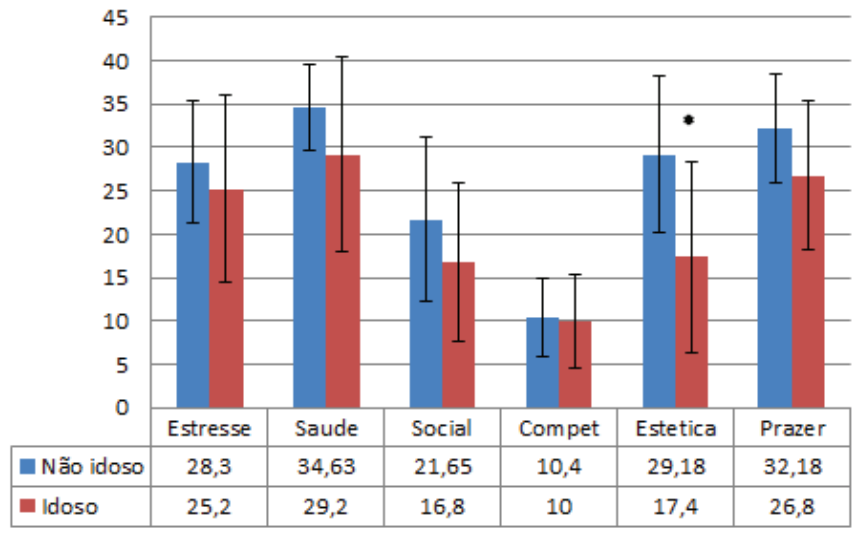

Figura 2. Comparação da motivação das mulheres idosas e não idosas
A figura 2 apresenta a comparação, na amostra feminina, da motivação das idosas e não idosas. A análise apresentou uma diferença significativa dentro da variável estética $(\mathrm{p}=0,01)$. A população feminina não idosa está mais motivada quando comparada as mulheres idosas. Porém, tanto as mulheres idosas quanto as não idosas apresentaram escores maiores em relação à saúde seguida pelo prazer.

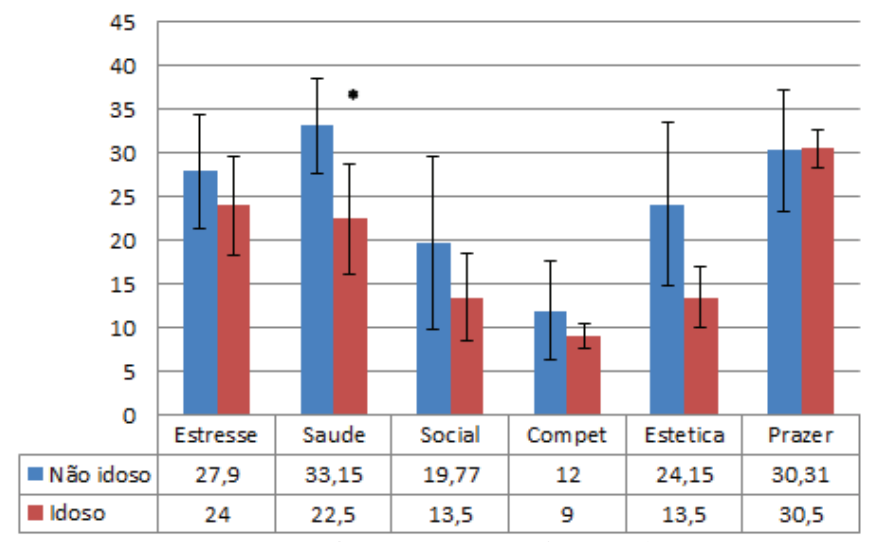

Figura 3. Comparação da motivação

dos homens idosos e adultos

A figura 3 apresenta a comparação da população masculina sobre a motivação entre os idosos e adultos. A análise estatística mostrou que a variável saúde apresentou uma diferença significativa entre idosos e adultos $(\mathrm{p}=0,025)$. Os homens adultos apresentam maior motivação nessa variável. Para os homens adultos a saúde seguida pelo prazer foram os domínios que apresentaram os maiores escores. E para os homens idosos o prazer seguido pelo estresse tiveram os maiores escores.

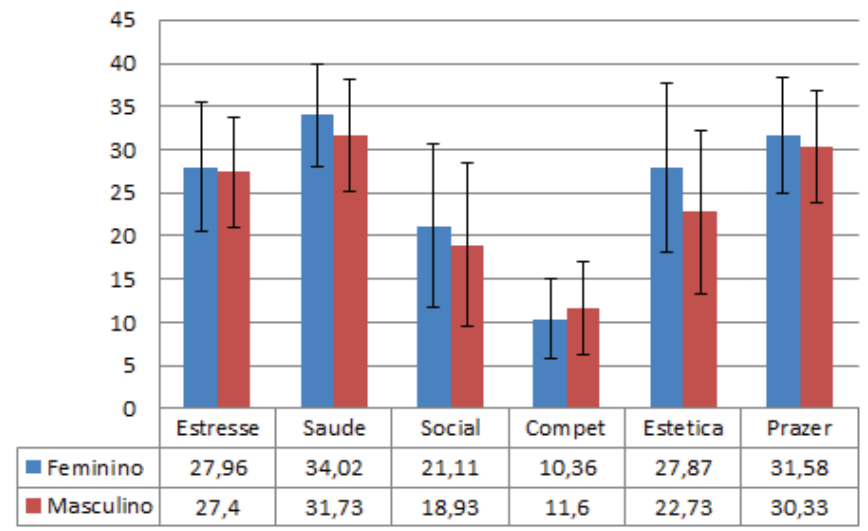

Figura 4. Comparação da motivação entre sexos

$\mathrm{Na}$ comparação da motivação entre os homens e as mulheres (figura 4) observou-se diferença significativa. Porém, tanto o público feminino quanto o masculino parecem se motivar para a prática através do domínio da saúde seguido pelo prazer. 


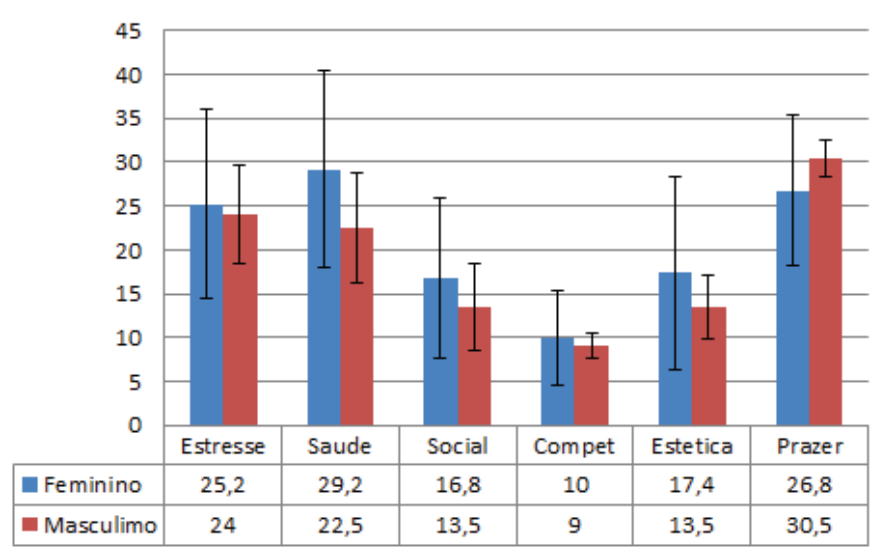

Figura 5. Comparação da motivação entre sexos dentro da população idosa

A análise dos aspectos motivacionais entre os homens e as mulheres idosos (figura 5) mostrou que não há diferenças significativas. Porém, homens e mulheres apresentam escores diferentes das variáveis motivacionais que envolvem a prática. Enquanto as mulheres idosas apresentaram escores maiores em relação ao domínio saúde seguidos pelo prazer, os escores dos homens idosos estavam mais altos em relação ao domínio prazer seguidos pelo estresse.

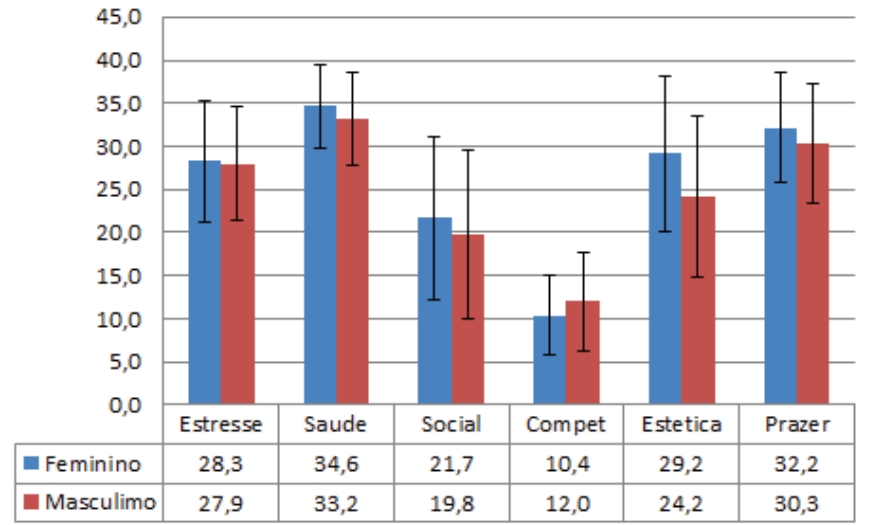

Figura 6. Comparação da motivação entre sexos dentro da população não idosa

A análise dos aspectos motivacionais entre os sexos feminino e masculino (figura 6), dentro da população de adultos. $\mathrm{Na}$ parcela da população não idosa, não houve diferença estatisticamente significativa e, em ambos os sexos, a motivação parece estar relacionada com saúde e prazer.

A relação da motivação entre os voluntários que apresentam menos de 6 meses de prática em comparação com os que possuem mais de 6 meses (figura 7), mostrou que no domínio da estética há diferença estatística significativa $(\mathrm{p}=0,026)$. O grupo com mais de 6 meses de prática se motiva mais em relação a essa variável em comparação com o grupo com menos de 6 meses de prática. Ambos os grupos parecem se motivar mais pela saúde, seguido pelo prazer.

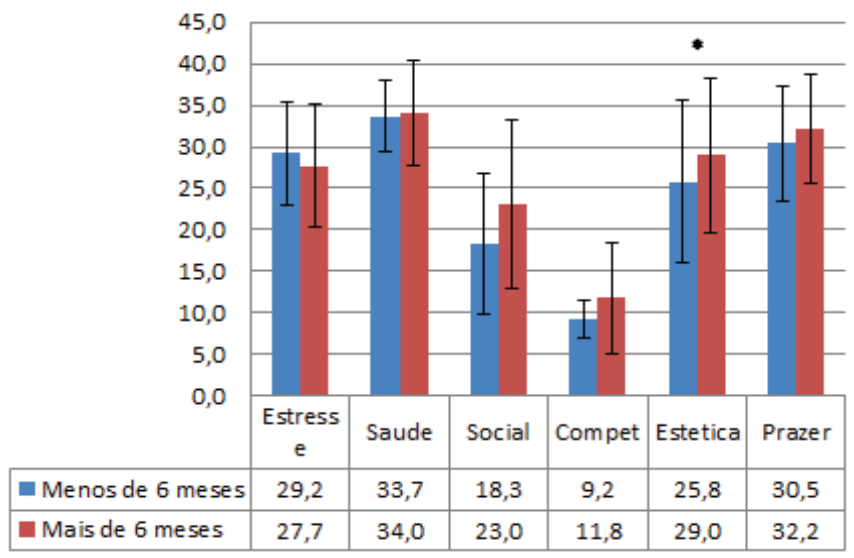

Figura 7. Comparação da motivação em relação ao tempo de prática.

Diante das análises, fica claro que tanto inter quanto intragrupos, todos os indivíduos praticantes de Pilates, parecem se motivar muito pouco para o domínio da competição em relação aos outros domínios apresentados.

\section{Discussão}

O objetivo do presente estudo foi investigar a motivação dos praticantes de Pilates, realizando uma comparação entre os participantes de acordo com sexo, faixa etária (adultos e idosos) e tempo de prática.

A faixa etária é uma variável que se mostra importante, pois está diretamente atrelada a autoimagem. Como visto nos achados deste estudo, mulheres adultas se motivam mais pelo domínio estética quando comparadas às idosas. Segundo Santos e Knijnik (2009), apesar da estética não ser o principal fator motivacional para a adesão de mulheres de 40 a 60 anos em exercícios físicos, elas se mostram bastante preocupadas em relação à autoimagem perante a sociedade. Houve um resultado semelhante ao presente trabalho. Os autores, Freitas, Santiago, Viana, Leão \& Frayre (2007), mostraram que os aspectos motivacionais que menos influenciam na adesão e manutenção de idosos em programas de prática de exercícios físicos, são: emagrecer e parecer mais jovem, que estão diretamente relacionados à variável estética.

Quando comparados homens adultos e idosos, a saúde foi determinante para homens adultos, este dado pode estar relacionado ao fato de que para este público o Pilates proporciona melhoras na força, função física 
e independência, melhoras importantes em relação à autonomia, benefícios psicossociais e elevada motivação para continuar a prática do exercício (Gaskell \& Williams, 2018). Os resultados encontrados corroboram novamente com Freitas, Santiago, Viana, Leão \& Frayre (2007), os quais encontraram que a maioria da sua amostra atribuiria a saúde como principal fator motivacional.

De forma geral, a população idosa do presente trabalho se mostrou mais motivada pelo domínio prazer. Porém, a diferença foi mínima em relação a valores absolutos quando comparado ao domínio saúde. Estudo em concordância com esses dados reporta que saúde, sociabilidade e prazer são os principais determinantes motivacionais entre idosos (Dantas, Andrada, Straatmann \& Lima, 2015). Além disso, muitos idosos procuram o método em busca da melhora da qualidade de vida ou manutenção da saúde (Costa, Schulz, Haas \& Loss, 2016).

Um fator importante a se destacar sobre a prática do método Pilates para melhorar o estado de saúde da população idosa e que pode influenciar em sua motivação é a liberação e indicação médica (Santos \& Knijnik, 2009). Especialmente quando apresentam problemas de saúde ou problemas articulares que, supostamente, os impossibilitam de fazer outro tipo de exercício. Este dado é reforçado pelo estudo de Gomes e Zazá (2009) realizado com uma amostra de mulheres idosas, cujo principal motivo de adesão aos exercícios físicos foi melhorar ou manter o estado de saúde.

O método Pilates é um forte aliado como exercício físico para a população idosa. Essa modalidade auxilia de forma positiva no equilíbrio, na flexibilidade, na força e na melhora da composição corporal. Além da melhoria na autonomia funcional e na redução do risco de quedas (Engers, Rombaldi, Portella \& Silva, 2016; Dantas, Andrada, Straatmann \& Lima, 2015; Bluhm, Silva, Geremia, Voser \& Lehnen, 2018). Fatores relacionados à saúde, sociabilidade e prazer foram determinantes motivacionais entre os idosos avaliados praticantes do Método Pilates (Dantas, Andrada, Straatmann \& Lima, 2015; Vilella, Zarceño \& Rosa, 2014). O estudo descreveu o uso de uma escala acadêmica de motivação, teste de atenção e teste completo de ansiedade de Spiebberge e descreveu que o Pilates é uma modalidade que melhora a ansiedade, atenção e desperta motivação para a prática (Aliev \& Memmedora, 2015). Sobre o fator ansiedade e estresse, estudo avaliou efeitos do método Pilates sobre a ansiedade e estresse e encontrou que quanto maior a prática semanal do método menor o índice de estresse e ansiedade, mostrando melhora em parâmetros psicológicos com a prática de Pilates (Herrera-Gutiérrez, Olmos-Soria, \& Brocal-Pérez, 2015).

A análise de todas as populações estudadas neste trabalho e as suas relações (de acordo com a faixa etária e o sexo), foi percebido que os grupos se motivam à prática principalmente pelo domínio da saúde seguida pelo prazer, porém apenas um grupo se manifestou de forma diferente. Segundo os dados, o grupo homens idosos se motivam mais em função do prazer seguido pelo domínio controle do estresse, isso pode ser atribuído ao fato de dentro da amostra haver apenas um sujeito com essas características. O estudo de Freitas, Santiago, Viana, Leão \& Frayre (2007), analisou aspectos motivacionais que influenciam a adesão e manutenção de idosos em programas de exercícios físicos. Na sua amostra havia uma quantidade superior de homens idosos, no total de 37 indivíduos. Os resultados encontrados foram um pouco diferentes do presente trabalho, onde esses idosos se motivam mais pela questão da saúde e variáveis que fazem parte do âmbito da saúde, seguida pelo prazer pela prática, o fator "reduzir o estresse" aparece apenas como fator de manutenção do exercício físico e ainda assim, aparece em quarto lugar na ordem de importância dos mesmos (Freitas, Santiago, Viana, Leão \& Frayre, 2007).

Sobre a prática de Pilates pelo público feminino, estudos revelam que a saúde é o principal motivo da procura pela prática corporal, seguido de qualidade de vida e bem-estar (Pereira, Liberali, Lopes, Cruz, Artaxo Neto, Viana \& Mota, 2013; Dantas, Andrada, Straatmann \& Lima, 2015). Balbinotti e Capozzoli (2008) realizaram um trabalho exploratório em academias de ginástica, onde investigaram a motivação para a prática de exercícios por este público e encontraram que a variável que mais motiva o público jovem adulto e meia idade seria a saúde, enquanto para o público mais jovem o fator que mais motiva a prática era o prazer, resultados esses que corroboram com os achados do presente estudo.

A comparação entre a motivação que leva a prática do Pilates em relação à prática de exercícios físicos em academia de ginásticas, aparece novamente o fator saúde como o principal motivo para adesão e prática. Crocetta, Viana \& Andrade (2010) encontraram a estética como fator de alta motivação para a prática em academias o que se assemelha aos resultados encontrados para a população não idosa do presente trabalho, porém essa variável apresentou um baixo nível de motivação para a população idosa. Tahara, Schwartz \& Silva (2003) encontraram resultados similares, tendo como sua amostra alunos até 24 anos de idade, observou-se que esse público se motiva 
mais por questões estéticas, seguido por fatores que levam a melhoria ou manutenção da qualidade de vida que está diretamente relacionado com a saúde.

Grande parte da população geral da amostra é muito motivada pelo fator saúde, isso pode estar diretamente relacionado com o grau de escolaridade dos praticantes, pois a maioria possui ensino superior e maior acesso à informação, portanto, maior possibilidade de obter o conhecimento acerca dos benefícios que o exercício físico proporciona, dentre eles a saúde. A partir da investigação do nível de exercício físico, condições de saúde e características sociodemográficas de mulheres idosas brasileiras, Mazo, Mota, Gonçalves \& Matos (2005) descobriram que, dentro da sua amostra, $87,5 \%$ da parcela que possuía mais de oito anos de estudo apresentavam-se mais ativas em relação aos demais participantes. Em contraponto, em relação à parcela dessa população com menor tempo de instrução, os números se apresentaram de forma diferente, onde havia uma porcentagem menor de idosas mais ativas em relação à parte que possuía mais oito anos de estudo.

Em relação ao tempo de prática da modalidade de exercícios propostos pelo método Pilates, assim como o restante das análises que foram feitas, tanto o grupo com mais de seis meses de prática quanto o grupo com menos de seis meses, apresentaram a motivação relacionada à saúde seguida pelo domínio do prazer. Porém o grupo com mais de seis meses de experiência na prática, apresentou uma motivação maior e estatisticamente significativa em relação ao domínio estética. Como a prática proporciona ganhos em diversos aspectos, como a força que está diretamente ligada à hipertrofia e resistência ligada à definição muscular, a explicação se dá pelo fato dos alunos com mais tempo de treinamento se motivem pelas modificações corporais que a prática pode propiciar (Gaskell \& Williams, 2018).

Apesar de o método Pilates não ser o mais indicado para o ganho de massa muscular, com o tempo pode vir sim a ocorrer estímulos hipertróficos, melhorando o perfil antropométrico corporal (Engers, Rombaldi, Portella \& Silva, 2016; Donzelli, Domenica, Cova, Galletti \& Giunta, 2006), modificando e tonificando o corpo do praticante, tornando-o mais motivado em relação ao domínio estética. No início dessa prática corporal, o aluno começa também a gerar um déficit energético diário, que no decorrer do tempo, pode auxiliar na diminuição do percentual de gordura total, diminuindo assim o peso total, auxiliando de forma positiva no padrão estético do indivíduo, melhorando também seus níveis motivacionais em relação ao domínio da estética (Bluhm, Silva, Geremia, Voser \& Lehnen, 2018; Donzelli, Domenica, Cova, Galletti \& Giunta, 2006).

Espera-se que esse estudo possa auxiliar os profissionais da área da Educação Física e Fisioterapia que trabalham com o Pilates. Também, contribuir com uma intervenção mais qualificada e eficiente que auxilie no alcance dos objetivos dos alunos e dos profissionais envolvidos na intervenção, controlando e divulgando o resultado dos seus trabalhos nos aspectos psicossociais sempre levando em consideração o interesse e satisfação do indivíduo. Dessa forma, o Pilates tomará novas proporções, pois deixará de ser apenas um método replicado para ser um método com resultados cientificamente comprovados e respeitado no campo científico.

\section{Considerações finais}

Pode-se concluir que fatores relacionados à saúde e prazer foram determinantes motivacionais entre a população estudada. Foram realizadas análises entre os grupos idosos e adultos comparando-os em relação ao sexo e ao tempo de prática. A população de mulheres adultas apresentou uma maior motivação em relação ao domínio estética quando comparada com as idosas. Os homens adultos apresentam uma maior motivação em relação à saúde quando comparados com os idosos. Os participantes com mais de seis meses de prática se motivam mais em relação a variável estética quando comparado com os que possuem menos de seis meses de prática. Sugere-se a realização de novos trabalhos que envolvam amostras mais expressivas, com maior número de indivíduos do sexo masculino e idosos praticantes do método Pilates. Além disso, espera-se que novos estudos com foco nos aspectos psicológicos e método Pilates sejam realizados, uma vez que a literatura brasileira é carente de estudos dessa natureza.

Entender os aspectos motivacionais relacionados à prática do Método Pilates pode contribuir para uma melhor intervenção diretamente associada à satisfação dos praticantes, uma vez que, se entendermos o que leva o indivíduo a se engajar na modalidade, podemos elaborar uma intervenção que considere esses aspectos, melhorando a qualidade do serviço, e consequentemente, a aderência.

O Pilates é uma prática que tem ganhado adeptos pelo mundo, porém existe uma tendência de se replicar práticas associadas ao método que, muitas vezes, são incompatíveis com as demandas dos alunos. Isso se torna 
ainda mais crítico se considerarmos os aspectos relacionados às demandas pessoais, pois quando se procura reproduzir um método, não se pode esquecer que quem o realiza são sujeitos carregados de individualidades e significados, se tornando indispensável considerar suas motivações e satisfação. A soma do ser de cada indivíduo e seu perfil vão te mostrar os caminhos a serem percorridos com a fundamentação do método. Respeitando a individualidade e motivação de cada um, será possível eliminar hábitos posturais errados e contribuir para uma melhor qualidade de vida através da contrologia.

\section{Referências}

Aliev, R.; \& Memmedora, K. (2015). Application of Z-Number based Modeling in Psychological Research. Computational Intelligence and Neuroscience. http:// dx.doi.org/10.1155/2015/760403.

Balbinotti, M. A. A., \& Campozzoli, C. J. (2008). Motivação à prática regular de atividade física: Um estudo exploratório com praticantes em academias de ginástica. Revista Brasileira de Educação Física e Esporte, São Paulo, 22(1), 63-80, jan./mar.

Barbosa, M. L. L. (2006). Propriedades métricas do inventário de motivação à prática regular de atividade física (IMPRAF-120), Coleção Ciências da Saúde: Ciências do Movimento Humano.

Barra, B. S., \& Araújo, W. B. (2017). O efeito do método Pilates no ganho da flexibilidade. 2017. 37f. Monografia - Faculdade de Ciências Aplicadas "Sagrado Coração" Unilinhares, Espírito Santo.

Branco, A. N. C., Miyamoto, G. C., Soliano, A. C. G., Farhat, H. A., Franco, K. F. M., \& Cabral, C. M. N. (2017). Comparação da satisfação, motivação, flexibilidade e dor muscular tardia entre método Pilates moderno e método Pilates instável. Fisioterapia Pesquisa, 24(4), 427-436. DOI: 10.1590/1809.2 950/17685224042017.

Bluhm, C. C., Silva, M. R., Geremia, J. M., Voser, R. C., \& Lehnen, A. M. (2018). Perfil do estilo de vida individual em sujeitos fisicamente inativos e praticantes de Pilates. Revista Brasileira de Prescrição e Fisiologia do Exercício, 12(79), Supl.2, 975-984. Jul/ Dez.

Costa, L. M. R., Schulz, A., Haas, A. N., \& Loss, J. (2016). Os efeitos do método Pilates aplicado à população idosa: Uma revisão integrativa. Revista Brasileira de Geriatria e Gerontologia, 19(4), 695-702, Rio de Janeiro.

Dantas, R., Andrada G. P., Straatmann, V. S., \& Lima, V. P. (2015). Fatores motivacionais de idosos praticantes do método Pilates. Revista Brasileira em Promoção da Saúde, 28(2), jan-mar.

Donzelli, S., Domenica, E. D., Cova, A. M., Galletti, R., Giunta, N. (2006). Two different techniques in the rehabilitation treatment of low back pain: a randomized controlled trial. Eura Medicophys. 42(3):205-10.

Engers, P. B., Rombaldi, A. J., Portella, E. G., \& Silva, M. C. (2016). Efeitos da prática de Pilates em idosos: uma revisão sistemática. Revista Brasileira de Reumatologia, 56(4), 352-365.

Feijó, O. G. (1998). Corpo e movimento: Psicologia para o esporte. 2. Ed. Rio de Janeiro: Shape.

Formighieri, F. L. (2012). Força abdominal de mulheres praticantes do método Pilates. Monografia - Centro de Desportos, Universidade Federal de Santa Catarina, Florianópolis, Santa Catarina.

Freitas, C. M. S. M., Santiago, M. de S., Viana, A. T., Leão, A. C., \& Frayre, C. (2007). Aspectos motivacionais que levam a adesão e manutenção de idosos a programas de exercícios físicos. Revista Brasileira de Cineantropometria \& Desempenho Humano, 9(1), 92-100.

Gaskell, L., \& Williams, A. E. (2018). A qualitative study of the experiences and perceptions of adults with chronic musculoskeletal condition following a 12 week Pilates Exercise programme. Musculoskeletal care, 7(1), 54-62. https://doi.org/10.1002/msc.1365

Gomes, K. V., \& Zazá, D. C. (2009). Motivos de adesão a prática de atividade física em idosas. Revista Brasileira de Atividade Física \& Saúde, 14(2).
Herrera-Gutiérrez, E., Olmos-Soria, M., \& Brocal-Pérez, D. (2015). Efectos psicológicos de la práctica del Método Pilates em uma muestra universitária. Anales de Psicologia, 31(3), octubre, 916-920.

Jesus, R, E., Cruz, T. M. C., Liberali, R., Artaxo Netto, M. I., Viana, H. B., \& Lopes, C. R. (2013). Perfil de qualidade de vida em praticantes de Pilates. Fisioterapia Brasil, 14(5), 370-375.

Legnani, R. F. S., Guedes, D. P., Legnani, E., Barbosa Filho, V. C., \& Campos, W. (2011). Fatores motivacionais associados à prática de exercício físico em estudantes universitários. Revista Brasileira Ciências Esporte, Florianópolis, 33(3), 761-772, jul./set.

Liposcki, D. B., Ribeiro, A. C. W., \& Schneider, R. H. (2016). Utilização do método Pilates: reabilitação e condicionamento físico. Fisioterapia Brasil, 17(1).

Liz, C. M., Crocetta, T. B., Viana, M. da S., \& Andrade, R. B. A. (2010). Aderência à prática de exercícios físicos em academias de ginástica. Motriz, Rio Claro, 16(1), 181-188, jan./mar.

Mazo, G. Z., Mota, J., Gonçalves, L. H. T., \& Matos, M. G. (2005). Nível de atividade física, condições de saúde e características sócio-demográficas de mulheres idosas brasileiras. Revista Portuguesa Ciências do Desporto, 5(2), 202-212.

Miranda, R., \& Bara Filho, M. G. (2008). Construindo um atleta vencedor: Uma abordagem psicofísica do esporte. Porto Alegre: Artmed.

OMS. Organização mundial da Saúde. (2017). Disponível em: http://www. who.int/en/ Acesso em: 03 de jul.

OMS. Physical activity. Fevereiro 2018. Disponível em: http://www.who.int/ mediacentre/factsheets/fs385/en/ Acesso em: 03 de jun. 2020.

Panelli, C., \& De Marco, A. (2006). Método Pilates de condicionamento do corpo. São Paulo: Phorte.

Pereira, E. C., Liberali, R., Lopes, C. R., Cruz, T. M. F., Artaxo Netto, M. I., Viana, H. B., \& Mota, G. R. (2013). Aderência de pessoa no Pilates solo. Fisioterapia Brasil, 14(1), janeiro/fevereiro.

Pinton, L. B., \& Franco A. C. S. F. (2007). Influência do método Pilates: uma proposta de atividade física. Pontifícia Universidade Católica de Campinas PUC, Campinas - SP - Brasil. Coleção Pesquisa em Educação Física, 5(1).

Reis, L. A., Mascarenhas, C. H. M., \& LYRA, J. E. (2011). Avaliação da qualidade de vida em idosos praticantes e não praticantes do Método Pilates. Ciência \& Desenvolvimento - Revista Eletrônica da FAINOR, 4(1).

Santos, S. C., \& Knijnik, J. D. (2009). Motivos de adesão à prática de atividade física na vida adulta intermediária. Revista Mackenzie de Educação Física e Esporte, 5(1).

Schossler, A., Valente, T. A., Bittencourt, D. C., \& Strassburger, M. J. (2013). Efeitos dos exercícios do método Pilates em pacientes com dor lombar crônica. Revista Contexto \& Saúde, 9(16), 37-41.

Silva, R, B, F., \& Guerino, M. R. (2019). Revisão Método Pilates: benefícios e aplicabilidade para melhorar a qualidade de vida. Fisioterapia Brasil, 20(2).

Tahara, A. K., Schwartz, G. M., \& Silva, K. A. (2003). Aderência e manutenção da prática de exercícios em academias. Revista brasileira Ciência e Movimento, 11(4), 7-12.

Vieira, D. N., Testa, D., Ruas, P. C., Salvini, T. F., Catai, A. M., \& Melo, R. C. (2017). The effects of 12 week Pilates inspired exercise training on functional performance in older women. A randomized clinical trial. Journal of Bodywork and movement Therapies, 21(2), 251-258. https://doi.org/10.1016/ jibmt.2016.06.010

Vilella, S. B., Zarceño, E. L., \& Rosa, M. A. S. (2014). Hay benefícios psicosociales por la práctica Pilates? Um análisis de la literatura cientifica. Cuadernos de Psicologia del Deporte, 14(3), 117-128.

Vigitel - Vigilância de fatores de risco e proteção para doenças por inquérito telefônico. (2017). Disponível em http://portalarquivos.saude.gov.br/images/ pdf/2017/abril/17/Vigitel.pdf. Acesso em: 20 de mai. 2017.

Zen, J. M., Rempel, C., \& Grave, M. T. Q. (2016). Qualidade de vida de praticantes de Pilates e de sedentários. ConScientiae Saúde, 15(4), 593-603.

Submetido em: $31-8-2020$

Aceito em:19-4-2021 